\title{
Endocrine and metabolic diurnal rhythms in young adult men born small vs appropriate for gestational age
}

\author{
Charlotte Brøns ${ }^{1,2, *}$, Pernille N Saltbæk ${ }^{1, *}$, Martin Friedrichsen ${ }^{1,2}$, Yan Chen ${ }^{1}$ \\ and Allan Vaag ${ }^{1,2}$ \\ 'Department of Endocrinology (Diabetes and Metabolism), Rigshospitalet, Copenhagen, Denmark and \\ ${ }^{2}$ Steno Diabetes Center, Gentofte, Denmark \\ *(C Brøns and P N Saltbæk contributed equally to this work)
}

\author{
Correspondence \\ should be addressed \\ to C Brøns \\ Email \\ charlotte.broens@regionh.dk
}

\begin{abstract}
Objective: Sleep disturbances and alterations of diurnal endocrine rhythms are associated with increased risk of type 2 diabetes (T2D). We previously showed that young men born small for gestational age (SGA) and with increased risk of T2D have elevated fat and decreased glucose oxidation rates during nighttime. In this study, we investigated whether SGA men have an altered diurnal profile of hormones, substrates and inflammatory markers implicated in T2D pathophysiology compared with matched individuals born appropriate for gestational age (AGA). Methods: We collected hourly blood samples for $24 \mathrm{~h}$, to measure levels of glucose, free fatty acids (FFA), triglycerides (TG), insulin, C-peptide, leptin, resistin, ghrelin, plasminogen activator inhibitor-1 (PAI-1), incretins (GLP-1 and GIP), and inflammatory markers (TNF- $\alpha$ and IL-6) in 13 young men born SGA and 11 young men born AGA.

Results: Repeated measurements analyses were used to analyze the diurnal variations and differences between groups. The SGA subjects had increased 24-h glucose $(P=0.03)$, glucagon $(P=0.03)$ and resistin $(P=0.003)$ levels with no difference in diurnal rhythms compared with AGA controls. We found significant diurnal variations in levels of blood glucose, plasma TG, FFA, insulin, C-peptide, GLP-1, GIP, leptin, visfatin, TNF- $\alpha$, IL-6 and PAI-1. The variation in FFA levels differed between the groups during the evening. Plasma ghrelin and glucagon levels did not display diurnal variations.

Conclusions: Young men born SGA exhibit elevated 24-h blood glucose, and plasma glucagon and resistin levels with no major differences in diurnal rhythms of these or other key metabolic hormones, substrates or inflammatory markers implicated in the origin of adiposity and T2D.
\end{abstract}

\section{Introduction}

Disruption of biological rhythms, as seen for example with night-shift work and sleep deficiency or a disruption of sleep in general, is associated with an increased risk of developing obesity and type 2 diabetes (T2D) (1, 2, $3,4)$. The development of $\mathrm{T} 2 \mathrm{D}$ is due to complicated interactions between genetic and pre- and postnatal environmental factors. There is mounting evidence supporting that impaired fetal growth, associated with
() 2016 European Society of Endocrinology Printed in Great Britain being small for gestational age (SGA), represents an independent risk factor for T2D (5).

We and others have shown that individuals born SGA have reduced adult height and increased abdominal obesity as well as numerous metabolic impairments such as reduced protein expression of key insulin signaling molecules in muscle and adipose tissue, increased lipolysis, hepatic and peripheral insulin resistance, and 
disproportionately reduced insulin secretion $(6,7,8,9)$. In strong support of the 'thrifty phenotype' hypothesis as proposed by Hales and Baker (10), we have previously shown that young SGA men exhibit decreased energy expenditure when subjected to 36 -h fasting (11). Furthermore, recent data suggest that young healthy SGA men may have an altered diurnal rhythm including diurnal variations in metabolic hormones compared with control individuals born appropriate for gestational age (AGA), since they exhibit pronounced differences in substrate oxidation rates in particular during nighttime $(12,13)$. A central clock located in the suprachiasmatic nucleus (SCN) in the hypothalamus, generating a circadian (24-h) rhythm, regulates most physiological functions. The SCN plays a key role in controlling daily blood glucose homeostasis and regulates a part of the metabolism during the day through neural and/or humeral signaling $(14,15)$. It is synchronized to the 24 -h day primarily by daily light-darkness cycles; however, for many central diurnal processes, it is the timing and feedback from nutrient intake that is the most important stimulus for synchronizing diurnal oscillators $(16,17)$. A disruption of endocrine rhythms may result in hormonal dysfunctions affecting glucose homeostasis, thereby influencing the risk of developing metabolic disease including T2D (15).

The primary aim of this study was to examine whether individuals born SGA, compared with matched control individuals born AGA, exhibit an altered metabolic profile with regard to selected key hormones, substrates and inflammatory markers involved in the glucose and lipid metabolism. It includes change in plasma insulin, C-peptide, glucagon, ghrelin, leptin, resistin and visfatin; incretins glucagon-like peptide-1 (GLP-1) and gastric inhibitory polypeptide (GIP); cytokines TNF- $\alpha$, IL- 6 and plasminogen activator inhibitor-1 (PAI-1) levels, as well as glucose, triglycerides (TG) and free fatty acid (FFA) levels. We hypothesized that altered diurnal variation in the selected key hormones and substrates could explain the disproportionally increased nocturnal fat oxidation and decreased glucose oxidation recently reported in young men born SGA $(12,13)$.

\section{Subjects and methods}

\section{Subjects}

The participants were recruited using data from the Danish Medical Birth Registry. All of them were males born singletons at term (Week 39-41) in Copenhagen County. Small for gestational age was defined as a birth weight in the lowest 10th percentile and AGA was defined as a birth weight between the 50th and 90th percentile. In total, 48 healthy Caucasian men were recruited for the study. To avoid major genetic confounding, subjects with a family history of diabetes in two generations were excluded. Further, participants having BMI $>30 \mathrm{~kg} / \mathrm{m}^{2}$, those performing physical activity for $\geq 10 \mathrm{~h}$ per week, and those taking medication that influences the outcome measures were excluded.

Blood samples from the first participant were hemolyzed upon addition of a protease inhibitor, thus reducing the number of participants to 47 . Furthermore, because of the instability of samples analyzed in the first batch, we chose to exclude all uncertain measures with unacceptable high coefficient of variation (CV) values defined a priori in this case to include $\mathrm{CV}$ values $>25 \%$ in the duplicate samples. Therefore, in an unbiased and blinded manner, data from 12 AGA and 11 SGA men were excluded, subsequently resulting in valid data from a total of 13 SGA and 11 AGA individuals.

Informed written consent was obtained from all subjects after fully explaining the purpose and nature of the study. The study protocol was approved by the Local Ethics Committee, and procedures were performed according to the principles of the Helsinki Declaration.

\section{Study protocol}

All subjects were asked to refrain from strenuous physical activity and from alcohol intake for three days before the examination. All meals, $10 \mathrm{MJ} /$ day in accordance with current recommendations (18), were provided to the participants for 3 days before and during examinations. The study activities were carried out over 3 days. The subjects were admitted to Steno Diabetes Center at 18:00 h the day before the examination. Height and weight were measured and body composition was assessed with dualenergy X-ray absorptiometry (DXA) scanning. Participants had to go to sleep at 23:00 $\mathrm{h}$.

On the day of examination, the participants exercised on a bike at $60 \%$ of their maximum pulse for $10 \mathrm{~min}$ in the morning and afternoon to imitate a regular day. Similar to the preceding days, three main meals and three snacks were served. Breakfast was served at 07:30 h, lunch at $12: 10 \mathrm{~h}$, dinner at $18: 10 \mathrm{~h}$, and snacks were served in between meals (Fig. 1). Coffee/tea was offered with breakfast, lunch, and afternoon snack, and a glass of milk and a sugar-free soft drink was offered for dinner. Water was allowed ad libitum. Besides the bike ride, quiet indoor activities, except sleeping, were allowed during daytime. 


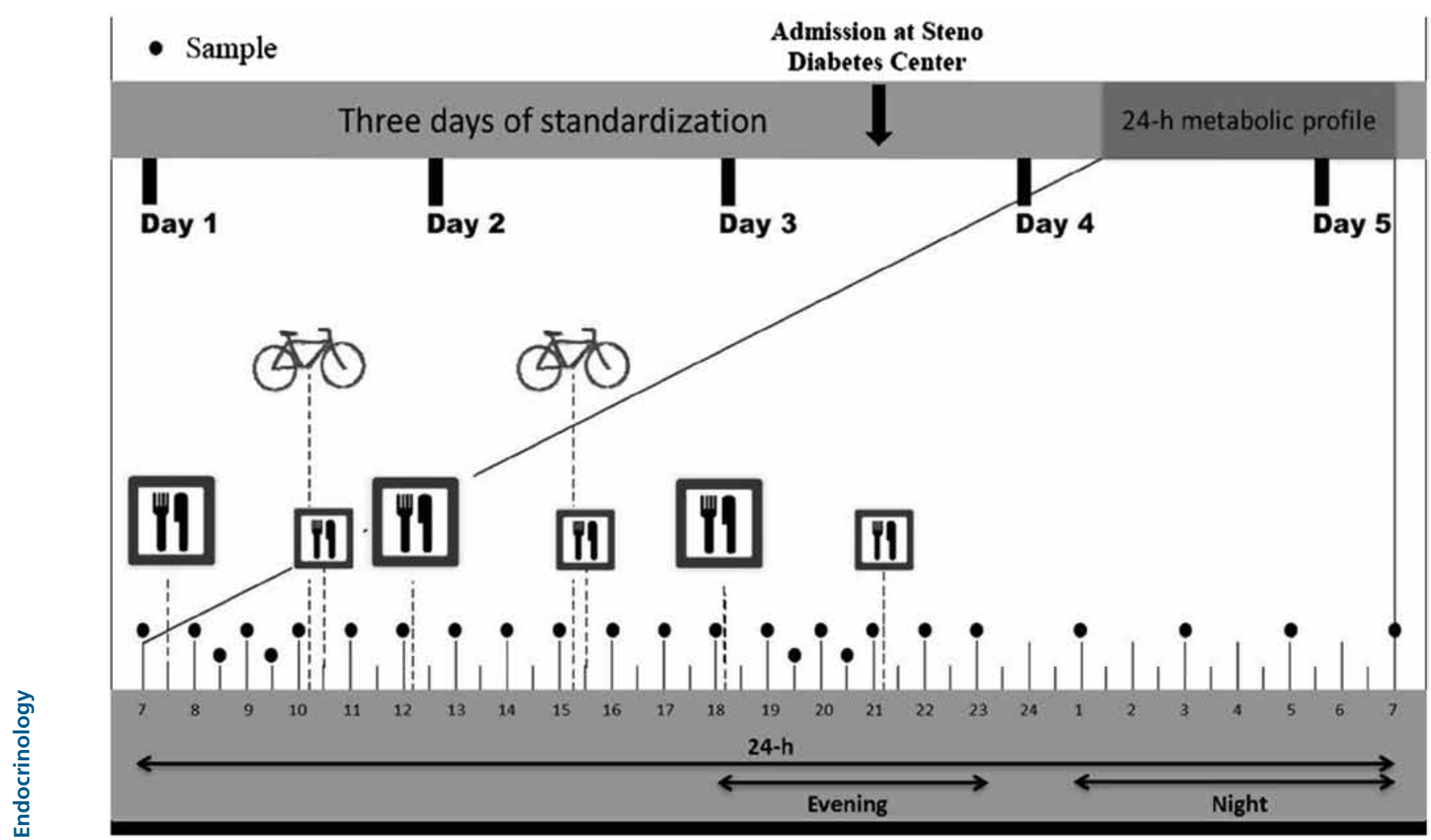

Figure 1

Overview of the study activities.

Smokers were offered sugar-free nicotine chewing gum. The participants were instructed to go to bed and sleep at 23:00 h, and the last blood sample was taken at 07:00 h the following morning. Hemoglobin levels were measured and iron supplements were handed out to all participants. After breakfast was served, the participants had to answer a questionnaire concerning normal sleep habits inspired by the Pittsburgh Sleep Quality Index (19), and they also had to score sleep quality during the night of the experiment. The participants were observed during the entire duration of the examination. The evening (postprandial) period was from 18:00 to 23:00 h and nighttime (sleep) period was from 01:00 to 07:00 h.

\section{4-h blood sampling}

Examinations started at 06:30h. An intravenous cannula (Venflon) was placed in the antecubital fossa in each arm through which 25 blood samples were collected from 07:00 h until 07:00 h the next day. Blood samples were taken every $30 \mathrm{~min}$ for $2 \mathrm{~h}$ after breakfast and dinner, every hour during the rest of the day, and every $2 \mathrm{~h}$ during nighttime. To avoid clotting of the Venflon, $2 \mathrm{~mL}$ saline was infused after sampling and $2 \mathrm{~mL}$ blood/saline was aspired and discarded before every sampling. All samples were immediately centrifuged and stored at $-80^{\circ} \mathrm{C}$ until analysis. A small flashlight was used for nighttime sampling, as the SCN is affected by light, especially at night. If participants woke up, they were instructed to keep quiet and turn their arm to a position at which the sample could be easily taken.

\section{Analysis of blood samples}

The Diabetes Pro Hu 12-plex assays from Luminex xMAP (Luminex, Austin, TX, USA) were used to measure plasma levels of insulin, C-peptide, glucagon, GLP-1, GIP, ghrelin, leptin, resistin, visfatin, IL-6, TNF- $\alpha$, and PAI-1. The assay had a precision intra-assay CV of $\leq 20 \%$, an inter-assay $\mathrm{CV}$ of $\leq 30 \%$, a cross-reactivity of $<0.5 \%$, and an accuracy (recovery range) of 70-130\% according to the manufacturer. The 12 analytes were measured simultaneously, and all procedures were performed according to the manufacturer's instructions. Thirty-six samples were loaded in duplicate on each plate along with a standard curve. After a 2-h incubation period, the 
plates were washed and analyzed using the Luminex 200 system.

Blood glucose levels were measured using an Ascensia Contour blood glucose meter (LifeScan, Milpitas, CA, USA) as it requires a low sample volume and has a CV less than $5 \%$. The same instrument was used on all subjects throughout the entire experimental period to avoid confounding. HbA1c was determined on the sampling day using high-pressure chromatography (Tosoh G7, Tokyo, Japan). Total plasma cholesterol, HDL cholesterol, and TG samples were analyzed using enzymatic colorimetric analysis on a Hitachi 912 (Roche Diagnostics). Plasma FFAs were analyzed using spectrometry on a Hitachi 912 (Roche Diagnostics). All above assays were analyzed collectively at Steno Diabetes Center, Gentofte, Denmark.

\section{Statistics}

The Bio-Plex Manager software (version 5.0; Bio-Rad, Hercules, CA, USA) used values from the standard dilution series to create a standard curve for each analyte; based on these curves, concentrations of the 12 analytes were determined using logistic regression. The standard curve was manually fitted by removing outliers at the low and/ or high end. The best curve fit was defined as one where the accuracy of all standard points were as close as possible to $100 \%$ of the expected concentration. When a standard curve was not optimal to detect the low concentration of a hormone, the standard curve from the other plate analyzed on the same day was used. Out-of-range (OOR) data as determined by the Bio-Plex Manager software were not included in the analyses. CV values were determined by the Bio-Plex Manager software for each analyte on a plate and used to assess intra-assay variance; if a duplicate sample had a CV $>25 \%$, it was considered an outlier and excluded. Data points outside the standard curve were also excluded.

We fitted a longitudinal linear mixed effects model with a random intercept and errors with an exponential covariance structure to account for correlation between measurements within subjects. The class variables group and time and their interaction were included as fixed effects. Based on the residual plots for normality, the response variables were log-transformed before analysis to make the residuals approximately normally distributed. Analyses were performed using Proc Mixed in SAS 9.3 (SAS Institute, Cary, NC, USA).

Repeated measurements were determined for 3 periods: the 24 -h period (07:00-07:00h), the evening (postprandial) period (18:00-23:00h), and the nighttime (sleep) period (01:00-07:00 h).

The total area under the curve (AUC) was calculated using the trapezoidal rule and the incremental AUC by subtracting the baseline area from AUC for the postprandial phase after dinner. Unpaired Student's $t$-test was used to detect differences between the birth weight groups, and the Kolmogorov-Smirnov test was used to check for normality. Non-normally distributed data were analyzed using nonparametric tests, whereas normally distributed data were analyzed using parametric tests. A $P$ value $\leq 0.05$ was considered significant. Data and figures in the text are given as mean \pm s.D., unless otherwise stated.

\section{Results}

\section{Subject characteristics}

As shown in Table 1, the SGA subjects had significantly lower birth weight than the AGA controls $(P=7.8 \mathrm{E}-11)$. There were no differences in age, height, weight, BMI, or blood pressure. The two groups did not differ with regard to fat distribution and there were no differences in either fasting blood glucose levels, $\mathrm{HbA1c}$, or fasting plasma concentrations of insulin, C-peptide, cholesterols, triglycerides, and FFAs. Quality of sleep did not differ between the two groups (AGA: $6.41 \pm 1.99$ vs SGA: $6.54 \pm 1.98, P=0.88$ ).

Table 1 Subject characteristics and baseline plasma concentrations for men born AGA and SGA. Data are presented as mean \pm S.D.

\begin{tabular}{|c|c|c|c|}
\hline & $\mathbf{A G A}(n=11)$ & SGA $(n=13)$ & $P$ value \\
\hline Birth weight (g) & $3802 \pm 163$ & $2778 \pm 251$ & $\overline{7.8 E-11}$ \\
\hline Age (year) & $23.2 \pm 0.9$ & $23.6 \pm 0.8$ & 0.21 \\
\hline Height $(\mathrm{cm})$ & $181 \pm 9$ & $178 \pm 4$ & 0.30 \\
\hline Weight (kg) & $77.1 \pm 7.3$ & $78.5 \pm 10.5$ & 0.71 \\
\hline BMI $\left(\mathrm{kg} / \mathrm{cm}^{2}\right)$ & $23.6 \pm 2.4$ & $24.8 \pm 2.7$ & 0.29 \\
\hline BP systolic (mgHb) & $124 \pm 9$ & $131 \pm 22$ & 0.26 \\
\hline BP diastolic (mgHb) & $76 \pm 11$ & $73 \pm 7$ & 0.70 \\
\hline \multicolumn{4}{|c|}{ Proportional fat distribution } \\
\hline Trunk/total fat mass $(\mathrm{g})$ & $0.44 \pm 0.05$ & $0.42 \pm 0.06$ & 0.51 \\
\hline Leg/total fat mass $(\mathrm{g})$ & $0.36 \pm 0.03$ & $0.38 \pm 0.04$ & 0.16 \\
\hline Trunk/leg fat mass (\%) & $0.91 \pm 0.15$ & $0.87 \pm 0.16$ & 0.53 \\
\hline \multicolumn{4}{|c|}{ Baseline plasma concentrations } \\
\hline Glucose (mmol/L) & $4.96 \pm 0.37$ & $5.07 \pm 0.35$ & 0.48 \\
\hline $\mathrm{HbA} 1 \mathrm{c}(\%)$ & $5.11 \pm 0.28$ & $5.13 \pm 0.22$ & 0.82 \\
\hline Insulin (pmol/L) & $283 \pm 122$ & $370 \pm 313$ & 0.40 \\
\hline C-peptide (pmol/L) & $317 \pm 90$ & $376 \pm 88$ & 0.12 \\
\hline Cholesterol (mmol/L) & $4.44 \pm 1.06$ & $4.14 \pm 0.63$ & 0.40 \\
\hline HDL cholesterol (mmol/L) & $1.13 \pm 0.19$ & $1.13 \pm 0.25$ & 0.99 \\
\hline Triglyceride (mmol/L) & $1.02 \pm 0.55$ & $1.05 \pm 0.44$ & 0.91 \\
\hline $\mathrm{FFA}(\mathrm{mmol} / \mathrm{L})$ & $473 \pm 258$ & $379 \pm 158$ & 0.29 \\
\hline
\end{tabular}

$B P$, blood pressure; FFA, free fatty acids. 


\section{Area under the curve}

The SGA individuals had a significantly higher 24-h $(P=0.02)$, evening (postprandial) $(P=0.01)$, and nighttime $(P=0.02)$ AUC for plasma resistin levels compared with the AGA controls (Table 2). The SGA individuals had a significantly higher postprandial $(P=0.03)$ as well as borderline increased 24-h $(P=0.06)$ AUC for plasma glucagon compared with the AGA control subjects. There were no other significant AUC differences between the two groups.

\section{Repeated measurements}

During the 24-h period, applying repeated measurements analyses revealed that the SGA and AGA group differed significantly with regard to blood glucose $(P=0.03)$, glucagon $(P=0.03)$, and resistin ( $P=0.003$ ) levels (Fig. 2 and Table 3). There was a significant difference in 24-h diurnal variation in blood glucose, plasma TG, FFA, C-peptide, GIP, leptin, IL-6, and PAI-1 (all $P<0.0001)$, insulin $(P=0.0007)$, GLP-1 $(P=0.03)$, visfatin $(P=0.007)$, and TNF- $\alpha(P=0.004)$ plasma concentrations (Figs 2 and 3; Table 3 ). There was no difference in the Time $\times$ Group interaction for any of the measured hormones or metabolites.

In the postprandial evening period (18:00-23:00 h), there was a difference between the two groups in blood glucose $(P=0.01)$, glucagon $(P=0.03)$, and resistin $(P=0.005)$ levels (Table 3$)$. Time exhibited an effect on the concentrations of blood glucose $(P=0.01)$; plasma TG, FFA, and C-peptide (all $P<0.0001)$; and GIP $(P=0.0002)$ during the postprandial period. There was a significant interaction between time and group for plasma FFA levels only ( $P=0.05)$ (Figs 2 and 3; Table 3).

During nighttime (01:00-07:00 h), there was a significant difference between the two groups in plasma resistin concentrations $(P=0.002)$. A variability in concentrations during the nighttime was found for blood glucose $(P=0.001)$, TG $(P=0.01)$, FFA $(P<0.0001)$, insulin $(P=0.04)$, C-peptide $(P<0.0001)$, GLP-1 $(P=0.005)$, GIP $(P<0.0001)$, leptin $(P=0.04)$, resistin $(P=0.002)$, visfatin $(P=0.003)$, TNF- $\alpha(P=0.006)$, IL-6 $(P=0.008)$, and PAI-1 $(P=0.0008)$ plasma concentrations. There was no difference in the Time $\times$ Group interaction for any of the hormones or metabolites.

\section{Discussion}

The aim of this study was to explore whether an altered metabolic diurnal rhythm could play a role in the link between being born SGA and risk of developing T2D. The

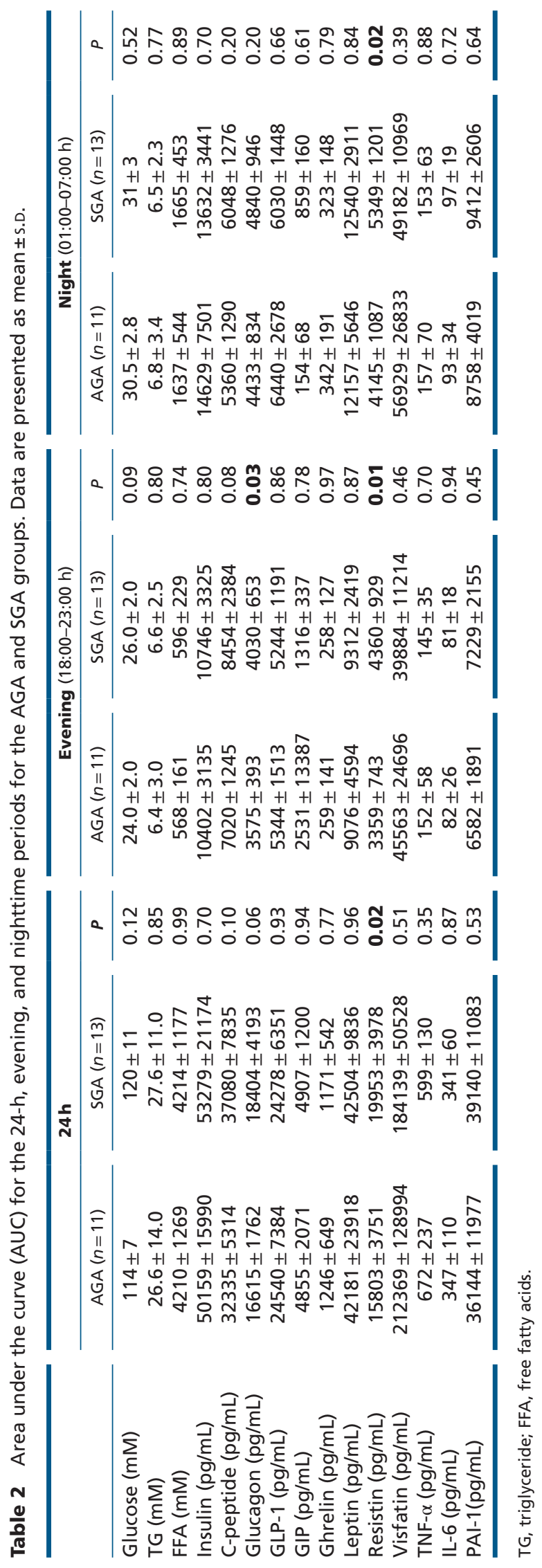

www.eje-online.org 
A

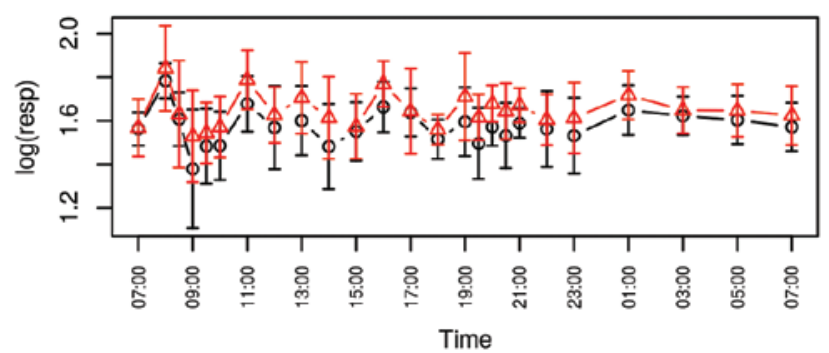

C

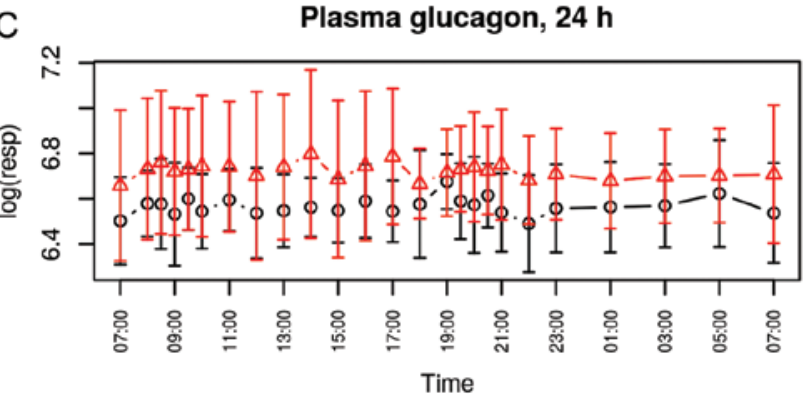

E

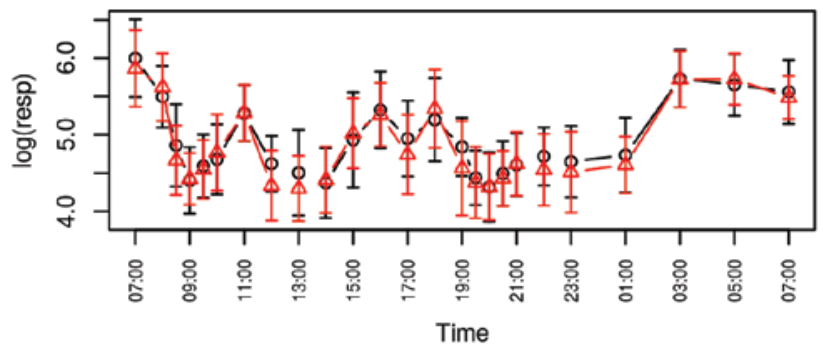

G

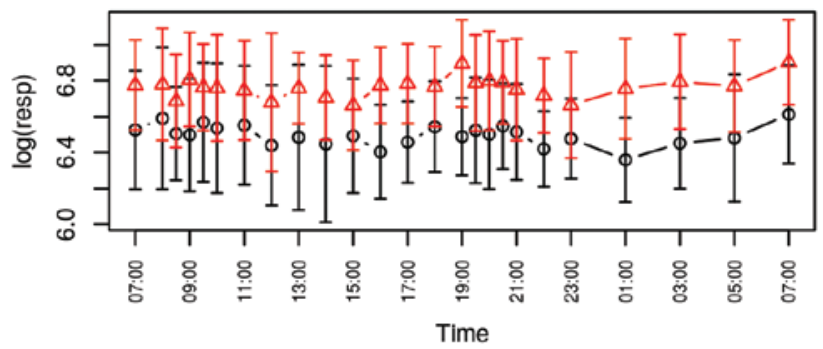

B

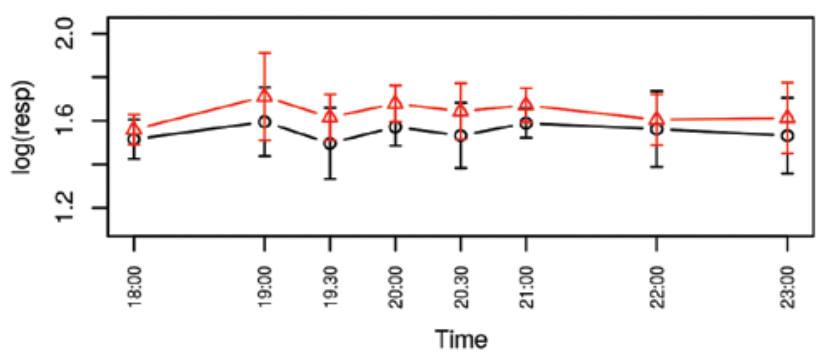

D

Plasma glucagon, evening (18-23)

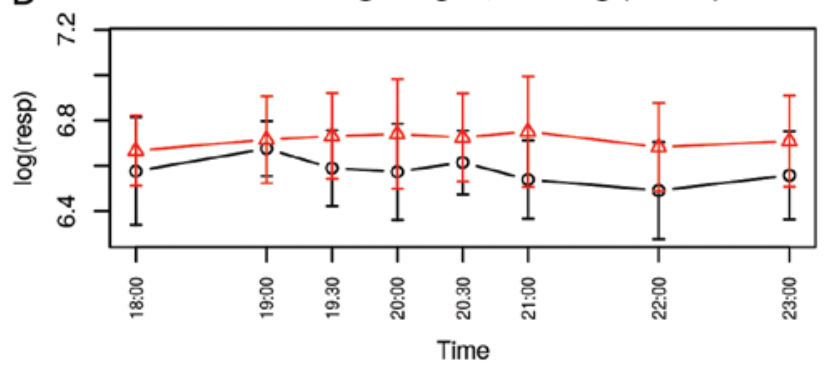

$\mathrm{F}$

Plasma free fatty acids, evening (18-23)

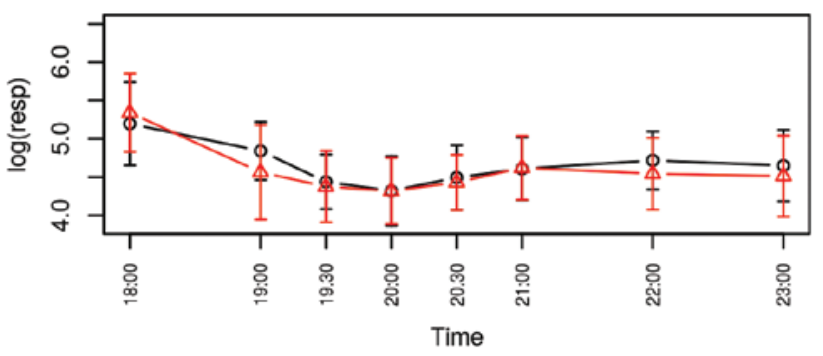

$\mathrm{H}$

Plasma resistin, night (01-07)

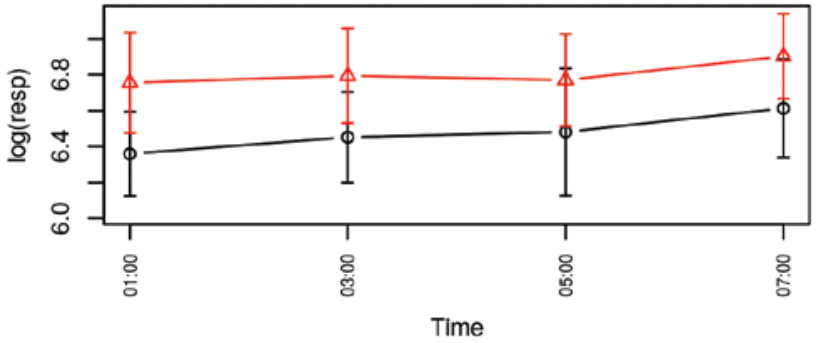

Figure 2

Selected diurnal rhythms of the SGA (red) and AGA (black) individuals for the $24 \mathrm{~h}$ and evening period respectively. (A and B) glucose, (C and D) glucagon, (E and F) FFA, (G and H) resistin.

experimental conditions in our study were designed to reflect general daily life conditions, and the participants were neither sleep-deprived nor exposed to forced dyssynchrony (manipulating with the 24 -h day) as seen in other studies $(16,20,21)$.

We did not find any statistical significant differences in the clinical baseline characteristics between the two groups as reported earlier $(6,7,22)$. However, this may be due to fewer study participants compared with our previous studies. Despite this, we were able to confirm the prior results showing significantly elevated blood glucose levels in the young SGA men compared with AGA controls in the 24-h repeated measurements analysis. This is in full agreement with the general consensus of SGA being a 
Table 3 Repeated measurements for group, time and time $\times$ group during the $24-h$, evening, and nighttime period. Data are presented as $P$ values and significant values are in bold.

\begin{tabular}{|c|c|c|c|c|c|c|c|c|c|}
\hline & \multicolumn{3}{|c|}{$24 h$} & \multicolumn{3}{|c|}{ Evening (18:00-23:00 h) } & \multicolumn{3}{|c|}{ Night $(01: 00-07: 00 \mathrm{~h})$} \\
\hline & Group & Time & Time $\times$ group & Group & Time & Time $\times$ group & Group & Time & Time $\times$ group \\
\hline Glucose (mM) & 0.03 & $<0.0001$ & 0.90 & 0.01 & 0.01 & 0.87 & 0.24 & 0.001 & 0.75 \\
\hline TG (mM) & 0.63 & $<0.0001$ & 0.13 & 0.72 & $<0.0001$ & 0.57 & 0.80 & 0.01 & 0.09 \\
\hline FFA (mM) & 0.61 & $<0.0001$ & 0.14 & 0.66 & $<0.0001$ & 0.05 & 0.77 & $<0.0001$ & 0.60 \\
\hline Insulin (pg/mL) & 0.84 & 0.0007 & 0.59 & 0.86 & 0.20 & 0.43 & 0.81 & 0.04 & 0.36 \\
\hline C-peptide (pg/mL) & 0.12 & $<0.0001$ & 0.58 & 0.07 & $<0.0001$ & 0.64 & 0.38 & $<0.0001$ & 0.12 \\
\hline Glucagon (pg/mL) & 0.03 & 0.43 & 0.75 & 0.03 & 0.41 & 0.36 & 0.12 & 0.62 & 0.59 \\
\hline GLP-1 (pg/mL) & 0.90 & 0.03 & 0.74 & 0.83 & 0.87 & 0.20 & 0.62 & 0.005 & 0.35 \\
\hline GIP (pg/mL) & 0.54 & $<0.0001$ & 0.56 & 0.59 & 0.0002 & 0.66 & 0.86 & $<0.0001$ & 0.06 \\
\hline Ghrelin (pg/mL) & 0.93 & 0.11 & 0.58 & 0.87 & 0.55 & 0.30 & 0.96 & 0.08 & 0.35 \\
\hline Leptin (pg/mL) & 0.54 & $<0.0001$ & 0.41 & 0.58 & 0.96 & 0.53 & 0.53 & 0.04 & 0.46 \\
\hline Resistin (pg/mL) & 0.003 & 0.59 & 0.80 & 0.005 & 0.10 & 0.25 & 0.002 & 0.002 & 0.64 \\
\hline Visfatin (pg/mL) & 0.94 & 0.007 & 0.81 & 0.62 & 0.98 & 0.51 & 0.71 & 0.003 & 0.75 \\
\hline TNF- $\alpha(p g / m L)$ & 0.47 & 0.004 & 0.61 & 0.93 & 0.78 & 0.06 & 0.69 & 0.006 & 0.54 \\
\hline IL-6 (pg/mL) & 0.73 & $<0.0001$ & 0.54 & 0.82 & 0.67 & 0.17 & 0.49 & 0.008 & 0.07 \\
\hline PAI-1 (pg/mL) & 0.73 & $<0.0001$ & 0.87 & 0.49 & 0.17 & 0.61 & 0.54 & 0.0008 & 0.08 \\
\hline
\end{tabular}

prediabetic state associated with increased risk of developing T2D (5). This finding illustrates the strength and statistical power of having multiple measurements over a 24-h period, in a limited number of subjects in contrast to having only one or few measurements in each subject in a larger sample size. Indeed, this also provides some assurance of an appropriate statistical power comparing the levels of other hormones and substrates between the two groups.

Interestingly, the increased concentration of blood glucose among the SGA subjects became even more significant in the evening, which is consistent with impaired glucose tolerance being more obvious at this time point (22). Using AUC comparisons and repeated measurements, we furthermore found that the individuals born SGA displayed elevated plasma concentrations of glucagon during the entire $24-\mathrm{h}$ period. Elevated plasma glucagon levels is a hallmark of T2D and may play a key role in the development of elevated hepatic glucose production rate and elevated fasting blood glucose levels in T2D as well as in prediabetic subjects (23). We, therefore, also speculate that the elevated plasma glucagon levels, documented for the first time in SGA subjects in this study, may contribute to the disproportionately elevated hepatic glucose production rate in SGA subjects as reported previously (24). The physiological explanation for why we are finding a higher concentration of glucagon in the SGA men may be due to an over-activity/expression of pancreatic $\alpha$-cells due to impaired $\beta$-cell function, or because of insulin resistance in the $\alpha$-cells, and thus a lack of negative feedback from insulin $(25,26,27)$.
Also, at all times, the SGA men displayed markedly elevated levels of plasma resistin compared with the controls born AGA. We did not find any variation in plasma resistin concentration during daytime and evening, but during the nighttime, plasma resistin concentrations increased toward the morning in both groups (Fig. $2 \mathrm{G}$ and $\mathrm{H}$ ). Human resistin is a proinflammatory cytokine expressed in and secreted by monocytic cells, and a recent metaanalysis provided evidence for an association between higher circulating resistin levels and increased mortality $(28,29)$. Furthermore, resistin gene expression is positively correlated with insulin resistance in humans. Serum resistin level is elevated in human obesity, which has been associated with the development of hepatic insulin resistance in mice $(30,31,32)$. Although the exact physiological and metabolic function(s) of resistin remains inconclusive in humans, it has been suggested to play a key role in the link between obesity and insulin resistance (33). In support of the current results, a study reported increased cord blood resistin levels in SGA newborns (34). Furthermore, young and healthy shift workers exhibit increased levels of resistin suggested to be associated with a misaligned diurnal rhythm and an early marker of the metabolic syndrome (35). Elevated plasma resistin levels have also been associated with increased inflammation including increased plasma IL-6 and TNF- $\alpha$ levels (36). Our finding of elevated plasma resistin levels in the face of normal plasma IL-6 and TNF- $\alpha$ level in prediabetic SGA men suggest that elevated plasma resistin may precede the development of overt inflammation with elevated inflammation markers. Plasma resistin could, therefore, potentially serve as an 

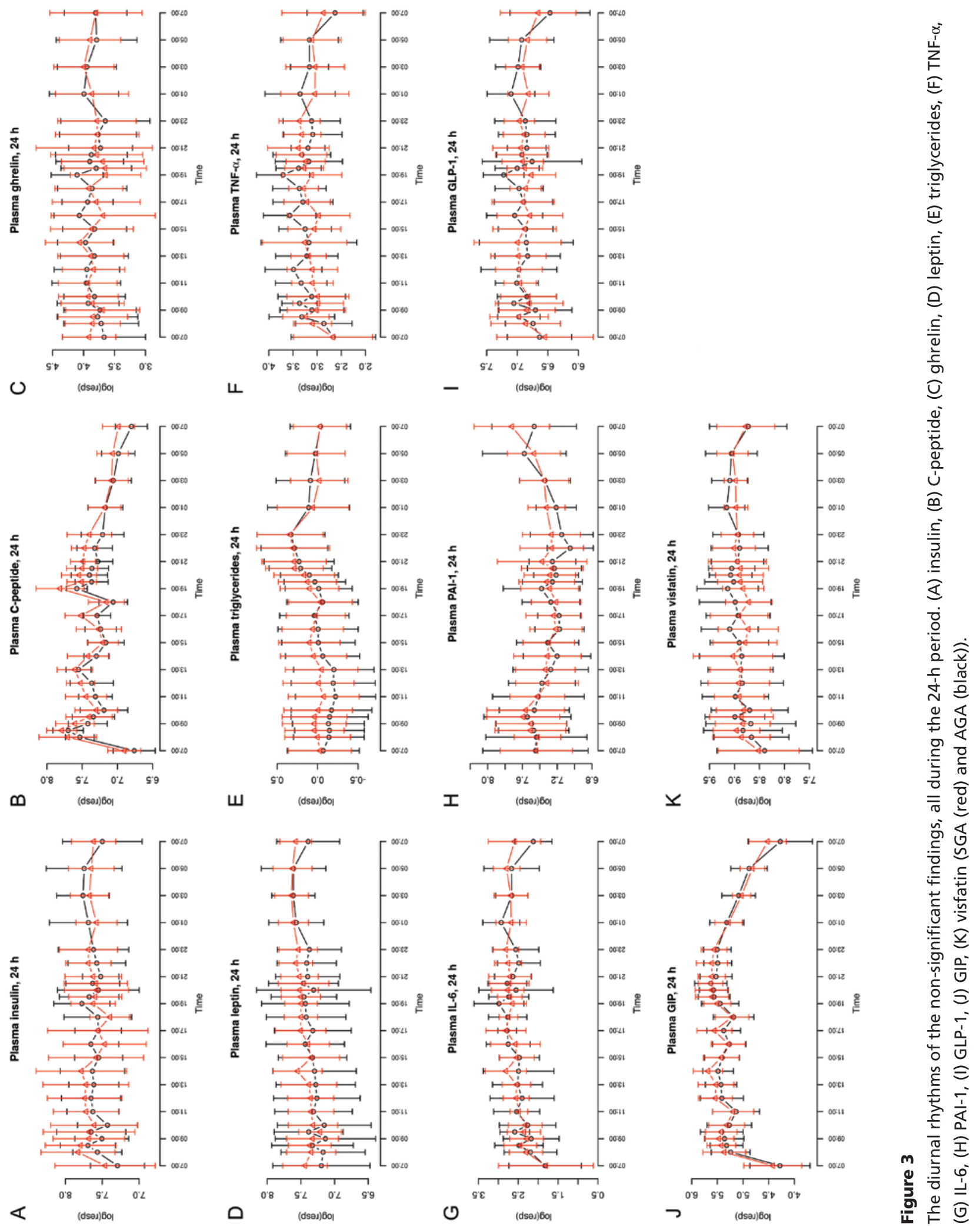

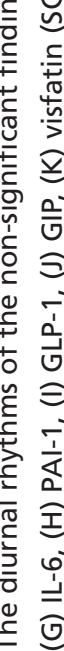


early marker of insulin resistance, T2D, and associated cardiometabolic disease in SGA individuals. However, further studies are needed to understand the origin, physiological function(s), and the potential adverse cardiometabolic consequences of the increased plasma resistin levels in human subjects born SGA.

Most of the hormones, substrates, and inflammatory markers showed a significant diurnal rhythmicity. Surprisingly, no significant diurnal variations in plasma glucagon, ghrelin, or resistin concentrations were observed during the 24-h period in any of the study groups, as would otherwise be expected in relation to food intake (37) (Figs 2C, G and 3C; Table 3). Similarly, the expected findings of diurnal rhythmicity of blood glucose, plasma TG, FFA insulin, and C-peptide levels support the dynamic variation in relation to food intake during the day and fasting during nighttime, as also shown in other studies $(15,16,22)$. The secretion of the gut incretin hormones GLP-1 and GIP is enhanced by food intake and decreased during fasting as also shown in the current study (38) (Fig. 3I and J; Table 3). Reduced plasma GLP-1 levels (39, 40) and impaired insulinotropic effect of the incretin hormone GIP are commonly seen in T2D patients $(38,41)$. We found, however, identical levels of both GLP-1 and GIP in the two groups, which are in agreement with previous studies showing similar fasting and postprandial incretin hormone levels in young and healthy SGA and AGA men $(24,42)$.

Ghrelin is a 'hunger' hormone and its levels are, therefore, highest right before a meal and lowest right after a meal (43). The meal-related regulation of plasma ghrelin is thought to be controlled by nutrient sensing either in the intestine or at postabsorptive sites. Interestingly, when applying repeated measurements, we found no diurnal fluctuation in plasma ghrelin concentrations, as would otherwise have been expected in response to food intake. However, when looking at the curve, it can be seen that, although not statistical significant, some fluctuation in plasma ghrelin levels is present in both groups, in particular during the daytime (Fig. 3C). One possible explanation for not finding large daily variations could be that the study participants were provided with meals and snacks throughout the day according to a set schedule, which may be the reason why the secretion of the "hunger' hormone was not stimulated. We previously studied fasting plasma ghrelin levels in SGA and AGA subjects and found no effect due to 5 days of high-fat overfeeding compared with a control diet between the two groups (24).

Leptin acts as the hormonal counterpart to ghrelin as a satiety hormone suppressing appetite. We found a $24-\mathrm{h}$ dynamic, as well as a nocturnal, rhythm in plasma leptin levels. The diurnal rhythm of leptin is also thought to be altered by meal timing and a nocturnal rise in leptin as has been shown previously, likely suppressing appetite while sleeping $(44,45)$. We previously showed that, in response to high-fat overfeeding, only AGA control subjects had significant increase in their plasma leptin, suggesting that the SGA individuals do not experience the same degree of appetite inhibition when exposed to overfeeding (24).

The diurnal variations in blood glucose, FFA, TG, insulin, C-peptide, GLP-1, and GIP levels are all well known and can be explained by meal-related fluctuations. However, the diurnal variations in plasma visfatin, TNF- $\alpha$, IL-6, or PAI-1 levels, as seen in both the SGA and AGA subjects in this group, may not be well known or easily explained in the context of meals. There was a small decrease in visfatin, TNF- $\alpha$, and IL- 6 concentrations (Fig. 3F, G and K), whereas PAI-1 concentration increased (Fig. $3 \mathrm{H}$ ) during nighttime. Visfatin is an adipokine that has been proposed to play a role in the development of obesity, insulin resistance, and/or T2D (46). Interestingly, people with sleep apnea syndrome have been reported to exhibit a disproportionately increased rise of plasma PAI-1 and visfatin levels from their nighttime nadir values to the peak day values (47). However, in this study, we were unable to demonstrate that SGA subjects showed any altered diurnal variation in plasma visfatin or PAI-1 levels compared with the AGA control subjects.

TNF- $\alpha$ and IL- 6 are key markers and mediators of systemic inflammation, which in turn have been implicated in the development of insulin resistance, T2D, cardiovascular disease, as well as in sleep disturbances (48, 49). However, we did not demonstrate any differences in plasma levels or in diurnal variations in plasma TNF- $\alpha$ or IL-6 levels in SGA subjects with a known increased risk of developing the above-mentioned diseases. The explanation for the diurnal variations in these inflammatory markers is unknown, but could be explained as being secondary to meal effects and perhaps the rise in plasma insulin levels in connection with meals $(50,51)$. However, further studies are needed to understand these variations over the day.

The link between T2D and diurnal disruption is primarily caused by extrinsic factors controlling the metabolism (i.e. change in sleep pattern and lack of sleep), which increases appetite, decreases leptin levels, and, therefore, leads to obesity and hence increased postprandial levels of glucose, increased insulin levels, and impaired glucose tolerance $(17,20)$. Thus, the increased risk of T2D due to 
diurnal disruption appears to be mediated primarily through weight gain. We did not find any differences between the diurnal rhythms of the SGA and the AGA (Table 3) groups that could explain the link between being born SGA and T2D, because the two groups were matched for weight.

We hypothesized that plasma FFA and/or TG levels might differ between the groups during nighttime. This hypothesis was based on the studies of 24-h substrate oxidation rates in SGA and AGA controls, the finding of increased nocturnal fat oxidation in SGA men $(12,13)$, as well as on our previously reported increased rate of lipolysis as determined using a stable isotope glycerol tracer in the fasting state among young men born SGA (7). However, in this study, no difference in plasma FFA levels between the groups was documented during nighttime. However, the evening differences between SGA and AGA lipid profiles with the FFA level falling after dinner and rising during nighttime, when the participants were fasting (Fig. 2E and F; Table 3), could support the notion of a disproportionately elevated rate of lipolysis driving the increased fat oxidation rate $(6,12,24)$.

Limitations of the study include low number of study participants and lack of information regarding catchup growth. Indeed, SGA subjects commonly display increased catch-up growth early in life, and increased catch-up growth per se has been proposed as an independent risk factor of cardiometabolic diseases including T2D later in life (52).

In conclusion, young and healthy adult men born SGA do not exhibit any major changes in hormonal or metabolic plasma diurnal rhythms compared with AGA control individuals. Increased plasma resistin and glucagon levels may contribute to the development of peripheral and hepatic insulin resistance and thereby elevated glucose levels in SGA subjects. More studies on human are needed to understand the origin and metabolic implications of the elevated plasma resistin level among the subjects born SGA.

\section{Declaration of interest}

The authors declare no conflict of interest that could be perceived as prejudicing the impartiality of the research reported.

\section{Funding}

This work was supported by the Danish Council for Independent Research - Medical Sciences (FSS), the Danish Council for Strategic Research, the Programme Commission on Food and Health (FøSU), the Danish Diabetes Association, the European Foundation for the Study of Diabetes (EFSD), the EU 6th Framework EXGENESIS Grant, and the Aase and Ejnar Danielsen Foundation.
Author contribution statement

C B designed the study, analyzed and interpreted data, and wrote the manuscript. $\mathrm{P} N$ interpreted data and drafted manuscript. $\mathrm{M} F$ and $\mathrm{Y} \mathrm{C}$ analyzed and interpreted data. A V designed the study and interpreted data. All authors critically revised the manuscript.

\section{Acknowledgements}

We wish to thank Camilla Larsen, Julie H Ziegler, and Julie W Iversen for completing the studies; Marianne Modest and Lars S Koch from Steno Diabetes Center for excellent support and sample analysis; and the kitchen personnel at Steno Diabetes Center for preparing all the food. Finally, we wish to thank all the young men for their participation in the study.

\section{References}

1 Kroenke CH, Spiegelman D, Manson J, Schernhammer ES, Colditz GA \& Kawachi I. Work characteristics and incidence of type 2 diabetes in women. American Journal of Epidemiology 2007165 175-183. (doi:10.1093/aje/kwj355)

2 Suwazono Y, Dochi M, Oishi M, Tanaka K, Kobayashi E \& Sakata K. Shiftwork and impaired glucose a 14-year cohort study on 7104 male workers. Chronobiology International 200926 926-941. (doi:10.1080/07420520903044422)

3 Meisinger C, Heier M \& Loewel H. Sleep disturbance as a predictor of type 2 diabetes mellitus in men and women from the general population. Diabetologia 200548 235-241. (doi:10.1007/s00125-0041634-x)

4 Gangwisch JE, Heymsfield SB, Boden-Albala B, Buijs RM, Kreier F, Pickering TG, Rundle AG, Zammit GK \& Malaspina D. Sleep duration as a risk factor for diabetes incidence in a large U.S. sample. Sleep 2007 30 1667-1673.

5 Vaag AA, Grunnet LG, Arora GP \& Brøns C. The thrifty phenotype hypothesis revisited. Diabetologia 201255 2085-2088. (doi:10.1007/ s00125-012-2589-y)

6 Brøns C, Jensen CB, Storgaard H, Alibegovic A, Jacobsen S, Nilsson E, Astrup A, Quistorff B \& Vaag A. Mitochondrial function in skeletal muscle is normal and unrelated to insulin action in young men born with low birth weight. Journal of Clinical Endocrinology and Metabolism 200893 3885-3892. (doi:10.1210/ jc.2008-0630)

7 Alibegovic AC, Højbjerre L, Sonne MP, van Hall G, Alsted TJ, Kiens B, Stallknecht B, Dela F \& Vaag A. Increased rate of whole body lipolysis before and after 9 days of bed rest in healthy young men born with low birth weight. American Journal of Physiology: Endocrinology and Metabolism 2010298 E555-E564. (doi:10.1152/ajpendo.00223.2009)

8 Jensen CB, Martin-Gronert MS, Storgaard H, Madsbad S, Vaag A \& Ozanne SE. Altered PI3-kinase/Akt signalling in skeletal muscle of young men with low birth weight. PLoS ONE 20083 e3738. (doi:10.1371/journal.pone.0003738)

9 Ozanne S, Jensen C, Tingey K, Martin-Gronert MS, Grunnet L, Brøns C, Storgaard H \& Vaag AA. Decreased protein levels of key insulin signalling molecules in adipose tissue from young men with a low birthweight - potential link to increased risk of diabetes? Diabetologia 200649 2993-2999. (doi:10.1007/s00125-006-0466-2)

10 Hales CN \& Barker DJP. Type 2 (non-insulin-dependent) diabetes mellitus: the thrifty phenotype hypothesis. Diabetologia 199235 595-601. (doi:10.1007/BF00400248)

11 Jørgensen SW, Brøns C, Bluck L, Hjort L, Færch K, Thankamony A, Gillberg L, Friedrichsen M, Dunger DB \& Vaag AA. Metabolic response to 36 hours of fasting in young men born small vs appropriate for gestational age. Diabetologia 201558 178-187. (doi:10.1007/s00125014-3406-6) 
12 Brøns C, Lilleøre SK, Jensen CB, Toubro S, Vaag A \& Astrup A. Increased nocturnal fat oxidation in young healthy men with low birth weight: Results from 24-h whole-body respiratory chamber measurements. Metabolism 201362 709-716. (doi:10.1016/j. metabol.2012.12.002)

13 Brøns C, Lilleore SK, Astrup A \& Vaag A. Disproportionately increased 24-h energy expenditure and fat oxidation in young men with low birth weight during a high-fat overfeeding challenge. European Journal of Nutrition 2015 In press. (doi:10.1007/s00394015-1018-7)

14 Green CB, Takahashi JS \& Bass J. The meter of metabolism. Cell 2008 134 728-742. (doi:10.1016/j.cell.2008.08.022)

15 Kalsbeek A, la Fleur S \& Fliers E. Circadian control of glucose metabolism. Molecular Metabolism 20143 372-383. (doi:10.1016/j. molmet.2014.03.002)

16 Patton DF \& Mistlberger RE. Circadian adaptations to meal neuroendocrine mechanisms. Frontiers in Neuroscience 20137185.

17 Reutrakul S \& Van CE. Interactions between sleep, circadian function, and glucose implications for risk and severity of diabetes. Annals of the New York Academy of Sciences 20141311 151-173. (doi:10.1111/ nyas.2014.1311.issue-1)

18 Becker W. [New Nordic nutrition recommendations 2004. Physical activity as important as good nourishing food]. Lakartidningen 2005 102 2757-2752.

19 Buysse DJ, Reynolds CF III, Monk TH, Berman SR \& Kupfer DJ. The Pittsburgh Sleep Quality Index: a new instrument for psychiatric practice and research. Psychiatry Research 198928 193-213. (doi:10.1016/0165-1781(89)90047-4)

20 Scheer FA, Hilton MF, Mantzoros CS \& Shea SA. Adverse metabolic and cardiovascular consequences of circadian misalignment. PNAS 2009106 4453-4458. (doi:10.1073/pnas.0808180106)

21 Gonnissen HK, Rutters F, Mazuy C, Martens EA, Adam TC \& Westerterp-Plantenga MS. Effect of a phase advance and phase delay of the 24-h cycle on energy metabolism, appetite, and related hormones. American Journal of Clinical Nutrition 201296 689-697. (doi:10.3945/ajcn.112.037192)

22 Sonnier T, Rood J, Gimble JM \& Peterson CM. Glycemic control is impaired in the evening in prediabetes through multiple diurnal rhythms. Journal of Diabetes and its Complications 201428 836-843. (doi:10.1016/j.jdiacomp.2014.04.001)

23 Faerch K, Borch-Johnsen K, Holst JJ \& Vaag A. Pathophysiology and aetiology of impaired fasting glycaemia and impaired glucose does it matter for prevention and treatment of type 2 diabetes? Diabetologia 200952 1714-1723. (doi:10.1007/s00125-009-1443-3)

24 Brøns C, Jacobsen S, Hiscock N, White A, Nilsson E, Dunger D, Astrup A, Quistorff B \& Vaag A. Effects of high-fat overfeeding on mitochondrial function, glucose and fat metabolism, and adipokine levels in low-birth-weight subjects. American Journal of Physiology: Endocrinology and Metabolism 2012302 E43-E51. (doi:10.1007/ s00125-012-2717-8)

25 Quesada I, Tuduri E, Ripoll C \& Nadal A. Physiology of the pancreatic alpha-cell and glucagon role in glucose homeostasis and diabetes. Journal of Endocrinology 2008199 5-19. (doi:10.1677/JOE08-0290)

26 Lee Y, Berglund ED, Yu X, Wang MY, Evans MR, Scherer PE, Holland WL, Charron MJ, Roth MG \& Unger RH. Hyperglycemia in rodent models of type 2 diabetes requires insulin-resistant alpha cells. PNAS 2014111 13217-13222. (doi:10.1073/pnas.1409638111)

27 Tsuchiyama N, Takamura T, Ando H, Sakurai M, Shimizu A, Kato K, Kurita S \& Kaneko S. Possible role of alpha-cell insulin resistance in exaggerated glucagon responses to arginine in type 2 diabetes. Diabetes Care 200730 2583-2587. (doi:10.2337/dc07-0066)

28 Patel L, Buckels AC, Kinghorn IJ, Murdock PR, Holbrook JD, Plumpton C, Macphee CH \& Smith SA. Resistin is expressed in human macrophages and directly regulated by PPAR gamma activators. Biochemical and Biophysical Research Communications 2003 300 472-476. (doi:10.1016/S0006-291X(02)02841-3)

29 Fontana A, Spadaro S, Copetti M, Spoto B, Salvemini L, Pizzini P, Frittitta L, Mallamaci F, Pellegrini F, Trischitta V et al. Association between resistin levels and all-cause and cardiovascular a new study and a systematic review and meta-analysis. PLOS ONE $2015 \mathbf{1 0}$ e0120419. (doi:10.1371/journal.pone.0120419)

30 McTernan CL, McTernan PG, Harte AL, Levick PL, Barnett AH \& Kumar S. Resistin, central obesity, and type 2 diabetes. Lancet 2002 359 46-47. (doi:10.1016/S0140-6736(02)07281-1)

31 Smith SR, Bai F, Charbonneau C, Janderova L \& Argyropoulos G. A promoter genotype and oxidative stress potentially link resistin to human insulin resistance. Diabetes 200352 1611-1618.

32 Muse ED, Obici S, Bhanot S, Monia BP, McKay RA, Rajala MW, Scherer PE \& Rossetti L. Role of resistin in diet-induced hepatic insulin resistance. Journal of Clinical Investigation 2004114 232-239. (doi:10.1172/JCI200421270)

33 Steppan CM, Bailey ST, Bhat S, Brown EJ, Banerjee RR, Wright CM, Patel HR, Ahima RS \& Lazar MA. The hormone resistin links obesity to diabetes. Nature 2001409 307-312. (doi:10.1038/35053000)

34 Farid SD, Najati N, Gharebaghi MM, Haghjo AG \& Ghojazadeh M. Resistin in cord blood of small for gestation age and appropriate for gestation age term neonates. Iranian Journal of Pediatrics 201323 659-663.

35 Burgueno A, Gemma C, Gianotti TF, Sookoian S \& Pirola CJ. Increased levels of resistin in rotating shift a potential mediator of cardiovascular risk associated with circadian misalignment. Atherosclerosis 2010210 625-629. (doi:10.1016/j. atherosclerosis.2009.12.032)

36 Tsiotra PC, Boutati E, Dimitriadis G \& Raptis SA. High insulin and leptin increase resistin and inflammatory cytokine production from human mononuclear cells. BioMed Research International 20132013 487081. (doi:10.1155/2013/487081)

37 Cummings DE, Purnell JQ, Frayo RS, Schmidova K, Wisse BE \& Weigle DS. A preprandial rise in plasma ghrelin levels suggests a role in meal initiation in humans. Diabetes 200150 1714-1719.

38 Holst JJ \& Orskov C. Incretin hormones - an update. Scandinavian Journal of Clinical \& Laboratory Investigation. Supplementum 2001234 $75-85$.

39 Vaag AA, Holst JJ, Volund A \& Beck-Nielsen H. Gut incretin hormones in identical twins discordant for non-insulin-dependent diabetes mellitus (NIDDM) - evidence for decreased glucagon-like peptide 1 secretion during oral glucose ingestion in NIDDM twins. European Journal of Endocrinology 1996135 425-432. (doi:10.1530/ eje.0.1350425)

40 Faerch K, Torekov SS, Vistisen D, Johansen NB, Witte DR, Jonsson A, Pedersen O, Hansen T, Lauritzen T, Sandbæk A et al. GLP-1 response to oral glucose is reduced in prediabetes, screen-detected type 2 diabetes, and obesity and influenced by the ADDITION-PRO Study. Diabetes 201564 2513-2525. (doi:10.2337/db14-1751)

41 Vilsboll T \& Holst JJ. Incretins, insulin secretion and type 2 diabetes mellitus. Diabetologia 200447 357-366. (doi:10.1007/s00125-0041342-6)

42 Schou JH, Pilgaard K, Vilsboll T, Jensen CB, Deacon CF, Holst JJ, Vølund A, Madsbad S \& Vaag AA. Normal secretion and action of the gut incretin hormones glucagon-like peptide- 1 and glucosedependent insulinotropic polypeptide in young men with low birth weight. Journal of Clinical Endocrinology and Metabolism 200590 4912-4919. (doi:10.1210/jc.2005-0382)

43 Williams DL \& Cummings DE. Regulation of ghrelin in physiologic and pathophysiologic states. Journal of Nutrition 2005135 1320-1325.

44 Sinha MK, Ohannesian JP, Heiman ML, Kriauciunas A, Stephens TW, Magosin S, Marco C \& Caro JF. Nocturnal rise of leptin in lean, obese, and non-insulin-dependent diabetes mellitus subjects. Journal of Clinical Investigation 199697 1344-1347. 
45 Schoeller DA, Cella LK, Sinha MK \& Caro JF. Entrainment of the diurnal rhythm of plasma leptin to meal timing. Journal of Clinical Investigation 1997100 1882-1887.

46 Chang YH, Chang DM, Lin KC, Shin SJ \& Lee YJ. Visfatin in overweight/obesity, type 2 diabetes mellitus, insulin resistance, metabolic syndrome and cardiovascular a meta-analysis and systemic review. Diabetes/Metabolism Research and Reviews 201127 515-527. (doi:10.1002/dmrr.v27.6)

47 Yano Y, Ohmori T, Shimada K, Sakata Y \& Kario K. Association of sleep onset of acute coronary syndrome with sleep apnea syndrome and abnormal diurnal variation of hemostasis and adipokine levels. Blood Coagulation and Fibrinolysis 201223 590-596. (doi:10.1097/ MBC.0b013e328355e885)

48 Gustafson B. Adipose tissue, inflammation and atherosclerosis. Journal of Atherosclerosis and Thrombosis 201017 332-341. (doi:10.5551/ jat.3939)
49 Izawa S, Miki K, Liu X \& Ogawa N. The diurnal patterns of salivary interleukin-6 and C-reactive protein in healthy young adults. Brain Behavior and Immunity 201327 38-41. (doi:10.1016/j. bbi.2012.07.001)

50 Dimitrov S, Besedovsky L, Born J \& Lange T. Differential acute effects of sleep on spontaneous and stimulated production of tumor necrosis factor in men. Brain Behavior and Immunity 201547 201-210. (doi:10.1016/j.bbi.2014.11.017)

51 Moller-Levet CS, Archer SN, Bucca G, Laing EE, Slak A, Kabiljo R, Lo JC, Santhi N, von Schantz M, Smith CP et al. Effects of insufficient sleep on circadian rhythmicity and expression amplitude of the human blood transcriptome. PNAS 2013110 E1132-E1141. (doi:10.1073/pnas.1217154110)

52 Jain V \& Singhal A. Catch up growth in low birth weight infants: striking a healthy balance. Reviews in Endocrine and Metabolic Disorders 201213 141-147. (doi:10.1007/s11154-012-9216-6)

Received 25 February 2016

Revised version received 15 April 2016

Accepted 20 April 2016 